\title{
Turning the Circadian Clock Slow: A New Function of Gaseous Signal $\mathrm{H}_{2} \mathrm{~S}$
}

\author{
Peng Zhang ${ }^{1}$, Lin $\mathrm{Na} \mathrm{Xu}^{1}$, Lan $\mathrm{Zhou}^{2}$ and Ji Min $\mathrm{Cao}^{2 *}$ \\ ${ }^{1}$ Key Laboratory of Medical Electrophysiology at Southwest Medical University, Ministry of Education, Medical \\ Electrophysiological Key Laboratory of Sichuan Province, Institute of Cardiovascular Research, Collaborative Innovation Center \\ for Prevention and Treatment of Cardiovascular Disease of Sichuan Province, Southwest Medical University, Luzhou, China
}

${ }^{2}$ Key Laboratory of Cellular Physiology at Shanxi Medical University, Ministry of Education, and the Department of Physiology, Shanxi Medical University, Taiyuan, China

*Corresponding author: Ji Min Cao, Key Laboratory of Cellular Physiology at Shanxi Medical University, Ministry of Education, and the Department of Physiology, Shanxi Medical University, Taiyuan, China

\section{ARTICLE INFO}

Received: 㓞 December 09, 2021

Published: 蔧 December 16, 2021

Citation: Peng Zhang, Lin $\mathrm{Na} \mathrm{Xu}$, Lan Zhou, Ji Min Cao. Turning the Circadian Clock Slow: A New Function of Gaseous Signal $\mathrm{H}_{2}$ S. Biomed J Sci \& Tech Res 40(4)2021. BJSTR. MS.ID.006478.

\section{ABSTRACT}

Abbreviations: NO: Nitric Oxide; CO: Carbonic Oxide; $\mathrm{H}_{2} \mathrm{~S}$ : Hydrogen Sulfide; CBS: Cystathionine Beta Synthase; CSE: Cystathionine Gamma Lyase; NaHS: Sodium Hydrosulfide; SCN: Suprachiasmatic Nucleus; LD: Light Dark; AOAA: AmionoOxyacetate; ACSF: Artificial Cerebrospinal Fluid; SD: Standard Deviation

\section{Short Communication}

Gaseous signal molecules, such as nitric oxide (NO), carbonic oxide (CO), and hydrogen sulfide $\left(\mathrm{H}_{2} \mathrm{~S}\right)$, widely exist in most tissues of the body, and exert important physiological functions. These molecules are endogenously synthesized and catabolized, can freely diffuse across the plasma membrane and regulate certain biological processes at physiological concentrations. $\mathrm{H}_{2} \mathrm{~S}$ is the latest discovered among the above three gaseous signal molecules. Endogenous $\mathrm{H}_{2} \mathrm{~S}$ is produced by the catalytic synthesis of L-cysteine hydrochloride by cystathionine beta synthase (CBS) or cystathionine gamma lyase (CSE). CBS and CSE belong to pyridoxal 5'-phosphate dependent enzymes, with differences in tissue distribution. CBS mainly distributes in the brain, especially in hippocampus and cerebellum. The $\mathrm{H}_{2} \mathrm{~S}$ concentration in rat serum was about $46 \mu \mathrm{mol} / \mathrm{L}$ and was $50-160 \mu \mathrm{mol} / \mathrm{L}$ in rat brain tissues. In vivo, endogenous $\mathrm{H}_{2} \mathrm{~S}$ mainly exists in two forms, i.e., free gas (about $1 / 3$ ) and sodium hydrosulfide (NaHS) (about 2/3). The two forms keep a dynamic equilibrium. As a $\mathrm{H}_{2} \mathrm{~S}$ donor, NaHS mainly exists in peripheral tissues, and maintains the stabilities of free $\mathrm{H}_{2} \mathrm{~S}$ level and $\mathrm{pH}$ in vivo. Studies on endogenous NO and CO have demonstrated that both of them play important roles in the regulation of circadian rhythm in organisms. It has also been proved that $\mathrm{H}_{2} \mathrm{~S}$ can change the expression patterns of clock genes such as Clock, Per2, Bmal1, and Rev-erb $\alpha$ by influencing the content ratio of NAD/NADH and the expression of Sirt1 in mice hepatocytes [1]. However, it is still unclear whether $\mathrm{H}_{2} \mathrm{~S}$ regulate the central circadian clock in vivo. The present study aimed to test the hypothesis that $\mathrm{H}_{2} \mathrm{~S}$ plays an important role in the regulation of central circadian clock located in the suprachiasmatic nucleus (SCN) by affecting the expression patterns of clock genes and clock-controlled genes. 
To test the hypothesis, we performed voluntary wheel running experiments in adult male C57BL6/J mice. Animals were first entrained to $12: 12 \mathrm{~h}$ light: dark (LD) circumstance for 7 days and then subjected to constant dark (DD) condition for experiments, with circadian time 12:00 (CT12) indicated as the beginning of subjective night. Data were analyzed using the ActiView Biological Rhythm Analysis software (MiniMeter Co., USA). To observe the effects of higher or lower $\mathrm{H}_{2} \mathrm{~S}$ on the circadian locomotor rhythm, intracerebroventricular (i.c.v.) injections of exogenous NaHS (50mmol/L, $4 \mathrm{ml}$ per injection) or amiono-oxyacetate (AOAA) (a
CBS inhibitor) (50mmol/L, $4 \mu \mathrm{l}$ per injection) were performed, respectively. AOAA was dissolved in artificial cerebrospinal fluid (ACSF) composed of (in mmol/L) $\mathrm{NaCl} 119, \mathrm{NaHCO}_{3} 26.2, \mathrm{KCl} 2.5$, $\mathrm{NaH}_{2} \mathrm{PO}_{4}$ 1.0, $\mathrm{MgCl}_{2}$ 1.3, glucose 10.0, pH 7.4. To check whether the SCN showed a normal light entrainment, short-time (15min) light exposure (200 lux) was performed. Period length (tau) was determined by the ActiView periodogram analysis. Phase shifts were defined as the difference between the predicted activity onset times and observed activity onset times in response to NaHS or light pulses.
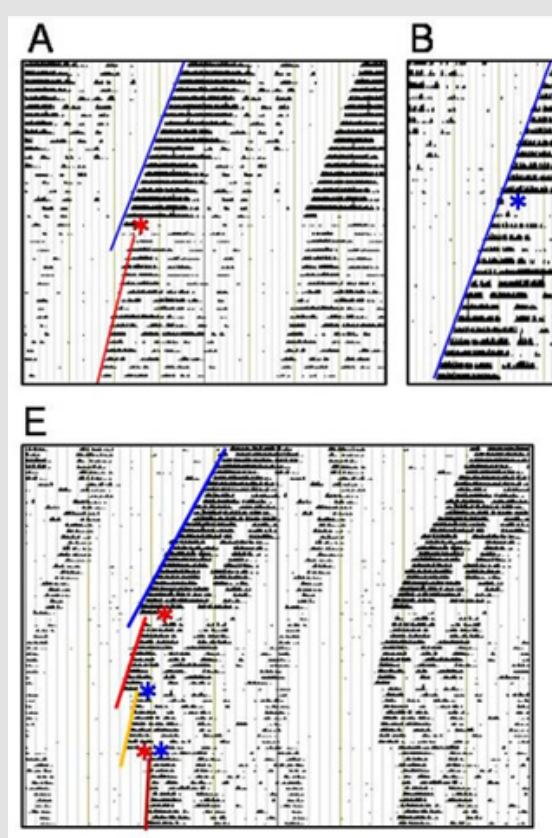

G $\quad$ CT2

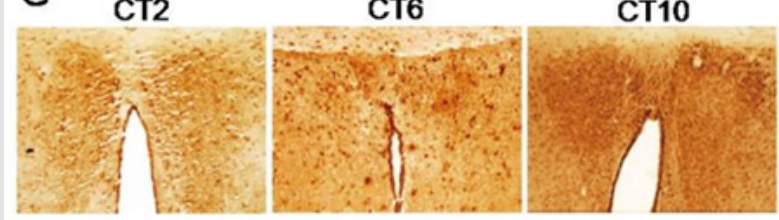

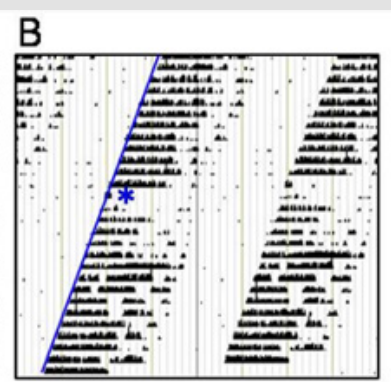

$\mathrm{F}$
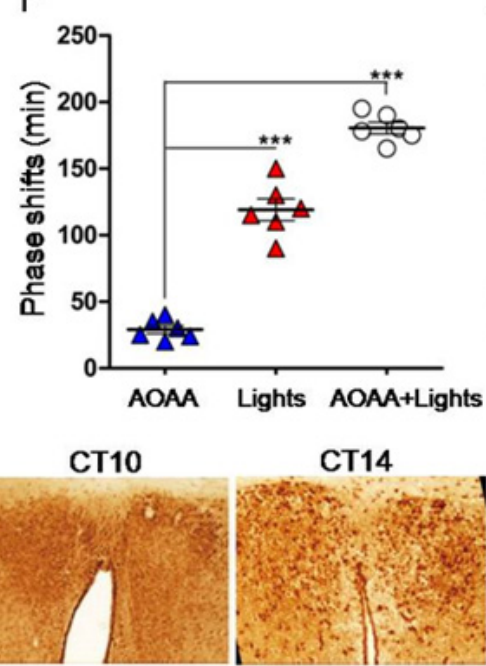

CT14
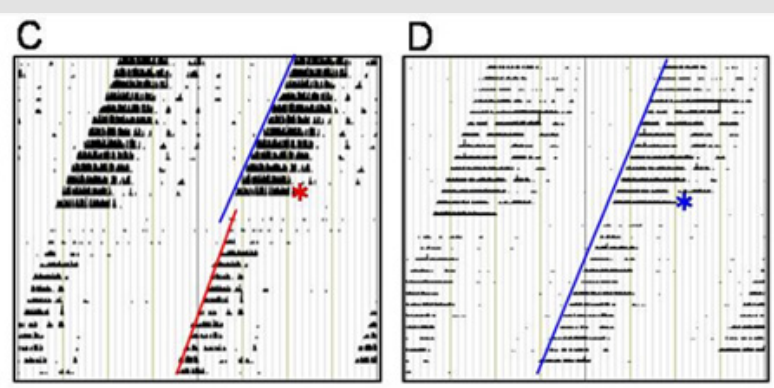

$\mathrm{H}$

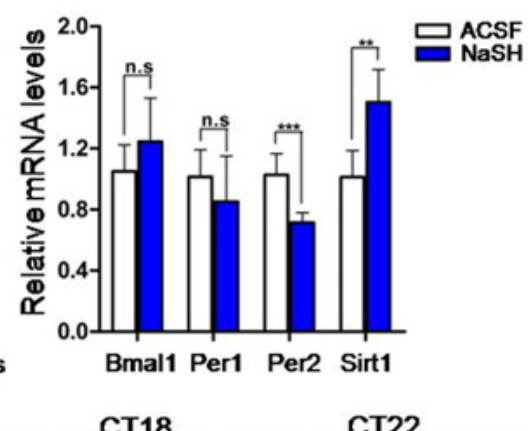

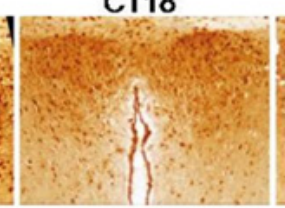

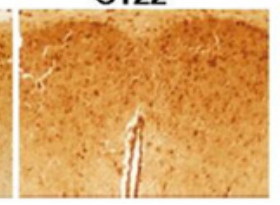

Figure 1: $\mathrm{H}_{2} \mathrm{~S}$ modulation on the circadian rhythm in mice.

A. Wheel running actograms showing that H2S i.c.v. injection at CT14 induced phase delay and circadian period prolongation.

B. ACSF i.c.v. injection at CT14 (control).

C. $\mathrm{H}_{2} \mathrm{~S}$ i.c.v. injection at CT20 also induced phase delay and circadian period prolongation.

D. ACSF i.c.v. injection at CT20.

E. Actograms showing phase shift and circadian period prolongation induced by i.c.v. injection of AOAA at CT14 (red asterisk), light exposure at CT15 (blue asterisk) and AOAA plus light exposure (red asterisk plus blue asterisk).

F. Statistical phase shifts at different conditions.

G. Immunohistochemical stains of SCN showing the robust circadian expression pattern of CBS.

H. RT-qPCR showing the mRNA levels of Bmal1, Per1, Per2 and Sirt1 in the SCN at different conditions.

After wheel running experiments, animals were decapitated under dim red light (4 lux), and SCN tissues were immediately harvested, liquid nitrogen frozen and total RNA were extracted as we previously reported [2]. Transcriptions of circadian genes, including Bmal1, Per1, Per2 and Sirt1, were examined by realtime quantitative PCR (RT-qPCR). Some SCN tissues were routinely 
developed, sectioned, and the protein expression of CBS was visualized by immunohistochemistry. Data were expressed as mean \pm standard deviation (SD). Student's t test or analysis of variance was used to perform the statistics where necessary. The criterion for statistical significance was $P<0.05$. Animal use protocol was in compliance with the guidelines for the Care and Use of Laboratory Animals (NIH, revised 2011). Results showed that bolus i.c.v. injection of NaHS at CT14 (early subjective night) induced significant phase delay of the circadian locomotor rhythm in mice by about $50 \pm 8 \mathrm{~min}$, and also obviously prolonged the circadian period length from $23.8 \mathrm{~h}$ to $24.3 \mathrm{~h}$ (Figure 1A), compared with the control ACSF injection at CT14 (Figure 1B). Bolus NaHS (i.c.v.) at CT20 (late subjective night) also caused a phase delay of $30 \pm$ $10 \mathrm{~min}$ (Figure $1 \mathrm{C}$ ) and a prolongation of period length from $23.8 \mathrm{~h}$ to 24h, compared with control ACSF injection at CT20 (Figure 1D). Above results showed that $\mathrm{NaHS}\left(\mathrm{H}_{2} \mathrm{~S}\right.$ donor) i.c.v. injection induced phase delay and prolongation of circadian period length, suggesting that $\mathrm{H}_{2} \mathrm{~S}$ could slow down the running of central circadian clock. We thus proposed that suppression of $\mathrm{H}_{2} \mathrm{~S}$ production by inhibiting CBS would lead to opposite changes of phase and period length. However, to our surprise, i.c.v. injection of AOAA at CT14 produced almost the same results as that induced by NaHS, i.e., phase delay $(30 \pm 10 \mathrm{~min})$ and period length prolongation, but the extents of phase shift and period prolongation were obviously less than that induced by NaHS (Figures $1 \mathrm{E} \& 1 \mathrm{~F}$ ).

To check whether NaHS i.c.v. injection would affect the light entrainment function of SCN, twelve days after bolus NaHS i.c.v. injection, we gave the mice a short-time (15min) light exposure at CT15. Results showed that short light exposure induced significant phase delay by about $120 \pm 20 \mathrm{~min}$ (Figures $1 \mathrm{E} \& 1 \mathrm{~F}$ ). This result indicates that the SCN responded well to light signal and was not damaged by NaHS i.c.v. injection. A combination of AOAA at CT14 and light exposure (CT15) led to further phase delay (180 $\pm 15 \mathrm{~min})$ and prolongation of circadian period length (Figures $1 \mathrm{E} \& 1 \mathrm{~F}$ ). As CBS is a $\mathrm{H}_{2} \mathrm{~S}$ synthetase, and $\mathrm{H}_{2} \mathrm{~S}$ donor NaHS affected the circadian rhythm as we observed, we thus examined whether CBS expression would show circadian rhythm, and whether inhibition of CBS would affect the expression of clock genes and clock-controlled gene Sirt1, in the SCN of mice. We detected the expression of CBS over 24 hours in mice SCN tissues by immunohistochemistry. SCN tissues were obtained at CT2, CT6, CT10, CT14, CT18 and CT22. CSB protein expression levels showed a robust circadian pattern with a cycle of nearly 24 hours (Figure 1G). The expression peak appeared at CT14, and the trough showed at CT2 (Figure 1G).

Because NaHS induced phase delay and period length prolongation as shown above, it is possible that NaHS may change the transcription of clock genes and clock-controlled gene Sirt1. We checked the mRNA levels of Bmal1, Per1, Per2 and Sirt1 before and $15 \mathrm{~min}$ after NaHS i.c.v. injection at CT14 by qRT-PCR. Results showed NaHS significantly decreased the mRNA levels of Per2 by 0.69-fold but increased the mRNA level of Sirt1 by 1.5 -fold, while NaHS did not affect the mRNA levels of Per1 and Bmal1 (Figure $1 \mathrm{H})$. To clarify the major findings of the present study, bolus i.c.v. injections of NaHS at CT14 and CT20, a way thought to increase the H2S level in the SCN, induced phase delay and period length prolongation of mice free-running locomotor rhythms; deactivating CBS by AOAA to reduce the $\mathrm{H}_{2} \mathrm{~S}$ level in the SCN exerted similar result as NaHS did; the used dosage of NaHS did not damage the light entrainment function of SCN; CBS expression in the SCN showed a pattern of circadian rhythm; NaHS i.c.v. injection at CT14 increased the protein expression of CBS and the transcription of Sirt1, decreased the transcription of Per2, but did not significantly affect the transcription of Bmal1 and Per1, in the SCN. These results suggest that $\mathrm{H}_{2} \mathrm{~S}$ slows down the running of circadian rhythm, or in other words, $\mathrm{H}_{2} \mathrm{~S}$ turns the central circadian clock slow. This study provides new evidences to support that $\mathrm{H}_{2} \mathrm{~S}$ takes a role in the regulation of central circadian clock. The circadian expression pattern of CBS in the SCN is at least partially associated with the regulatory function of $\mathrm{H}_{2} \mathrm{~S}$ on the central clock, and PER2 and SIRT1 may take major roles in the function of $\mathrm{H}_{2} \mathrm{~S}$.

We noticed that either increasing the $\mathrm{H}_{2} \mathrm{~S}$ level by NaHS or decreasing the $\mathrm{H}_{2} \mathrm{~S}$ level by CBS inhibitor induced the same results, i.e., phase delay and period length prolongation, only that the extent of NaHS-induced phase delay was greater than that caused by AOAA. Similar contradictory phenomena have also been observed in other studies. For example, Luo, et al. [3] demonstrated that both overexpression and deletion of miR-279 disrupted the circadian rhythm in Drosophila. We recently also found that either elevation or reduction of the microRNA-17-5p level in mice SCN shortened the free-running period length [4]. This unconventional regulatory algorithm deserves further investigations both in mechanism and significance. $\mathrm{H}_{2} \mathrm{~S}$ has been indicated having an anti-aging effect. It is still unknown whether $\mathrm{H}_{2} \mathrm{~S}$ exerts the anti-aging effect through changing the circadian rhythm. Some studies have shown that the free-running periods are shortened in senescent C57BL/6 mice, DBA mice, rats and hamsters, although some contradictory results were also reported. We show here that $\mathrm{H}_{2} \mathrm{~S}$ induced phase delay and free-running period prolongation in mice, and decreased Per2 mRNA level but increased Sirt1 mRNA level in the SCN. It is possible that $\mathrm{H}_{2} \mathrm{~S}$ exerts the anti-aging effect by prolonging the endogenous cycles of circadian rhythm via promoting the expression of Sirt1, because Sirt1 is considered a gene of longevity, and $\mathrm{H}_{2} \mathrm{~S}$ can prevent cell senescence through SIRT1 activation. SIRT1 regulates the expression of clock genes by PER2 acetylation [5]. Higher SIRT1 accelerates the degradation of PER2 protein, thereby promoting the transcription of Clock/Bmal1. This regulatory signaling pathway may explain our finding that exogenous $\mathrm{H} 2 \mathrm{~S}$ increased the expression of Sirt1 and decreased the expression of Per2. 
A question may be raised as whether exogenous NaHS (i.c.v.) would damage the function of SCN. The physiological concentration of $\mathrm{H}_{2} \mathrm{~S}$ in mouse brain is about $50-160 \mu \mathrm{mol} / \mathrm{L}$, but the exact transient local $\mathrm{H}_{2} \mathrm{~S}$ levels in the SCN are unknown. The concentration of NaHS used in the present study was $50 \mathrm{mmol} / \mathrm{l}$ (i.c.v. $4 \mathrm{ml}$ per injection). This NaHS concentration looked much higher than the $\mathrm{H}_{2} \mathrm{~S}$ level of natural brain, but the actual acting $\mathrm{H}_{2} \mathrm{~S}$ level in the SCN should be much lower than the used NaHS concentration if considering the dilution and diffusion of $\mathrm{NaHS} / \mathrm{H}_{2} \mathrm{~S}$ in the cerebrospinal fluid and brain tissues including SCN. We showed that the used NaHS concentration did not affect the light entrainment function of SCN, suggesting that SCN, or including other parts of mouse brain, is quite tolerant to NaHS/H2S. This finding suggests safety of relatively higher $\mathrm{H}_{2} \mathrm{~S}$ in the treatment of related brain diseases. In conclusion, $\mathrm{H}_{2} \mathrm{~S}$ can induce phase delay and circadian period length prolongation by affecting the expression patterns of clock genes and clock-controlled genes at SCN. This study suggests $\mathrm{H}_{2} \mathrm{~S}$ is regulator of central circadian clock.

\section{Acknowledgment}

This work was supported by NSFC grants (31471126 and 82170523 to J.C.), Sichuan Science and Technology Program

\section{ISSN: 2574-1241}

DOI: $10.26717 /$ BJSTR.2021.40.006478

Ji Min Cao. Biomed J Sci \& Tech Res

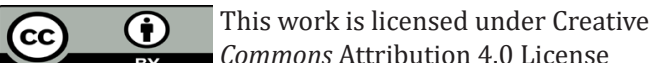

Submission Link: https://biomedres.us/submit-manuscript.php
(2019YJ0472 to P.Z.), and Luzhou Science and Technology Program (2018LZXNYD-ZK44 to P.Z.).

\section{Conflict of Interests}

The authors declare that they have no conflict of interest in this work.

\section{References}

1. Shang Z, Lu C, Chen S, Hua L, Qian R (2012) Effect of $\mathrm{H}_{2} \mathrm{~S}$ on the circadian rhythm of mouse hepatocytes. Lipids Health Dis 11: 23.

2. Zhou L, Gao Q, Nie M, Gu JL, Hao W, et al. (2016) Degeneration and energy shortage in the suprachiasmatic nucleus underlies the circadian rhythm disturbance in ApoE-/- mice: implications for Alzheimer's disease. Sci Rep 6: 36335 .

3. Luo W, Sehgal A (2012) microRNA-279 acts through the JAK/STAT pathway to regulate circadian behavioral output in Drosophila. Cell 148(4): 765-779.

4. Gao Q, Zhou L, Yang SY, Cao JM (2016) A novel role of microRNA 17-5p in the modulation of circadian rhythm. Sci Rep 6: 30070.

5. Asher G, Gatfield D, Stratmann M, Reinke H, Dibner C, et al. (2008) SIRT1 regulates circadian clock gene expression through PER2 deacetylation. Cell 134(2): 317-328.

$\begin{array}{ll}\text { BIOMEDICAL } & \text { Assets of Publishing with us } \\ \text { RESEARCHES } & \text { - Global archiving of articles } \\ \text { - Immediate, unrestricted online access }\end{array}$

